\title{
Reconstruction of Einstein-Aether Gravity from other Modified Gravity Models
}

\author{
Chayan Ranjit ${ }^{1 *}$, and Ujjal Debnath ${ }^{2 \dagger}$ \\ ${ }^{1}$ Department of Mathematics, Seacom Engineering College, Howrah - 711 302, India. \\ ${ }^{2}$ Department of Mathematics, Indian Institute of Engineering Science and Technology, Shibpur, Howrah-711 103, India.
}

(Dated: August 13, 2021)

\begin{abstract}
We briefly describe the modified Friedmann equations for Einstein-Aether gravity theory and we find the effective density and pressure. The purpose of our present work is to reconstruction of Einstein-Aether Gravity from other modified gravities like $f(T), f(R), f(G), f(R, T)$ and $f(R, G)$ and check its viability. The scale factor is chosen in power law form. The free function $F(K)$ for Einstein-Aether gravity (where $K$ is proportional to $H^{2}$ ) have been found in terms for $K$ by the correspondence between Einstein-Aether gravity and other modified gravities and the nature of $F(K)$ vs $K$ have been shown graphically for every cases. Finally, we analyzed the stability of each reconstructed Einstein-Aether gravity model.
\end{abstract}

PACS numbers: 04.50.Kd, 95.36.+x, 98.80.Cq, 98.80.-k

\section{INTRODUCTION}

Recently different observational study of the luminosity type Ia supernovae (SNIa) [1-4] and Cosmic Microwave Background (CMB) [5-9] radiation strongly indicate that our universe is currently expanding with an acceleration. There are many candidates which are considered as the main responsible for this expanding scenario. Dark energy (DE) [10 13] is one of them. There are different exotic types of dark energy with negative pressure. It can be separated by depending on their equation of state (EOS). When EOS is $-1<\omega<-1 / 3$, they are called quintessence type DE and when $\omega<-1$, they are called phantom type DE. There are some other type of dark energies model which can cross the phantom divide $\omega=-1$ from both sides and are called quintom type DE. Another idea to describe such acceleration scenarios is understood by imposing a concept of modification of gravity for an alternative candidate of dark energy. This type of model provides very natural gravitational alternative for exotic matter. This type of gravity models are predicted by string/M-theory. Modify Gravity models describe the phantom or non-phantom or quintom phase of the universe by without introducing negative kinetic term of dark energies. It gives a natural description about the early time inflation then transition from deceleration to late time acceleration. As a rival of General Relativity, some form of the theory of modified gravity are now very important for present research scenario while there are some rigid constraints in modify gravity theory. Cosmic acceleration of Dark energy models can also be explained by modifying in Ricci curvature $R$ of Lagrangian. Several form of Ricci curvature have been considered by the different researcher $14,15,17,18,45$ to explain the accelerating phase of the Universe. Remarkable results are obtained by some work of Nojiri et al [19, 20] in which they considered the modified Lagrangian as $R+R^{m}+R^{-m}$ and as a result they obtained an inflation at an early stage and also a late time of accelerated expansion. These result lead to open new window of gravity dependent research methodology. The most popular modified models of gravity include $f(R)$ gravity (where $R$ represent the Ricci Scalar Curvature) 21 23], $f(T)$ gravity (where $T$ is the torsion scalar) [24 26], $f(G)$ gravity (where $G$ represents the Gauss-Bonnet invariant) [27, 29], $f(R, T)$ gravity [30, 31], $f(R, G)$ gravity [32, 33] and many more.

Recently, reconstruction between different dark energy models become a very popular scenario among the researchers in Cosmological study. In 2008, Setare et al. 34 investigated the cosmological implications of the correspondence of Holographic dark energy model and Gauss-Bonnet dark energy model. This leads to a explanation of the accelerated expansion of the universe by imposing specific constraints. Liu et al [35] discussed the New Agegraphic Dark Energy (NADE) model in the framework of the Brans-Dicke theory with the help of EoS and deceleration parameters and as a result they showed that the EoS parameter has a quintom-like behavior for that model and it indicates the accelerated expansion of the universe. The NADE in $f(R)$ gravity model has been discussed in [36] where it is find that there may exist a phantom-like universe. Also in 37] the NADE model is correspond with Horava-Lifshitz gravity which indicates that the accelerated expanding universe is consistent with cosmological obser-

\footnotetext{
* chayanranjit@gmail.com

$\dagger$ ujjaldebnath@yahoo.com
} 
vations. The reconstruction of entropy-corrected Holographic Dark Energy (ECHDE) in the $f(G)$ gravity also been investigated to explain the expanding universe with acceleration in 38]. Recently the HDE model in the framework of the $f(G)$ gravity has been discussed in [39] and the different phenomenon for the accelerating universe are explained.

Motivated by the work of Debnath [40], we reconstructed the Einstein-Aether gravity models from the modified gravities like $f(T), f(R), f(G), f(R, T)$ and $f(R, G)$ by assuming a power law solution of the scale factor $\left(a=a_{0} t^{n}\right)$ separately. For this purpose, we first briefly explained the Einstein-Aether gravity theory by modifying the EinsteinHilbert action in section II and with the help of the modified Friedmann equations, we evaluated the effective density and pressure for Einstein-Aether gravity sector. After that in Sections III-VI, we have shown the correspondence between Einstein-Aether gravity and other modified gravities $f(T), f(R), f(G), f(R, T)$ and $f(R, G)$ and where we have reconstructed the unknown function $F(K)$ in term of $K$ and analyzed the nature of $F(K)$ in graphically. In Section VIII, we analyzed the stability of each reconstructed Einstein-Aether Gravity model. Finally, we described some cosmological implications of these reconstructed models in section IX.

\section{MODIFIED FRIEDMANN EQUATIONS IN EINSTEIN-AETHER GRAVITY THEORY}

Einstein-Aether Gravity Theory is basically a generalization of General Relativity(GR). The theory describes that the spacetime is coupled with both a metric and a dynamical unit timelike vector field named Aether. As it has a preferred reference frame and hence it violates Lorentz invariant. The action of the Einstein-Aether gravity theory with the normal Einstein-Hilbert part action can be written in the form [41-43]

$$
S=\int d^{4} x \sqrt{-g}\left[\frac{R}{16 \pi G}+\mathcal{L}_{E A}+\mathcal{L}_{m}\right]
$$

where $\mathcal{L}_{E A}$ is the vector field Lagrangian density while $\mathcal{L}_{m}$ denotes the Lagrangian density for all other matter fields. The Lagrangian density for the Einstein-Aether gravity is given by [42, 43]:

$$
\begin{gathered}
\mathcal{L}_{E A}=\frac{M^{2}}{16 \pi G} F(K)+\frac{1}{16 \pi G} \lambda\left(A^{a} A_{a}+1\right), \\
K=M^{-2}\left(c_{1} g^{a b} g_{c d}+c_{2} \delta_{c}^{a} \delta_{d}^{b}+c_{3} \delta_{d}^{a} \delta_{c}^{b}\right) \nabla_{a} A^{c} \nabla_{b} A^{d}
\end{gathered}
$$

where $c_{i}$ are dimensionless constants, $M$ is the coupling constant, $\lambda$ is a Lagrangian multiplier and $F(K)$ ia an arbitrary function of $K$. From (11), we get the Einstein's field equations

$$
G_{a b}=T_{a b}^{E A}+8 \pi G T_{a b}^{m}
$$

Here $T_{a b}^{m}$ is the energy momentum tensor for matter field and $T_{a b}^{E A}$ is the energy momentum tensor for the vector field and they are respectively given as follows: [43]

$$
T_{a b}^{m}=\left(\rho_{m}+p_{m}\right) u_{a} u_{b}+p_{m} g_{a b}
$$

where $\rho_{m}$ and $p_{m}$ are respectively the energy density and pressure of matter and $u_{a}=(1,0,0,0)$ is the fluid 4 -velocity vector and

$$
T_{a b}^{E A}=\frac{1}{2} \nabla_{d}\left[\left(J_{(a}{ }^{d} A_{b)}-J_{(a}^{d} A_{b)}-J_{(a b)} A^{d}\right) F^{\prime}\right]-Y_{(a b)} F^{\prime}+\frac{1}{2} g_{a b} M^{2} F+\lambda A_{a} A_{b}
$$

with

$$
Y_{a b}=-c_{1}\left[\left(\nabla_{d} A_{a}\right)\left(\nabla^{d} A_{b}\right)-\left(\nabla_{a} A_{d}\right)\left(\nabla_{b} A^{d}\right)\right]
$$

where the subscript $(a b)$ means symmetric with respect to the indices involved and $A^{a}=(1,0,0,0)$ is non-vanishing time-like unit vector satisfying $A^{a} A_{a}=-1$.

Now we consider the Friedmann-Robertson-Walker (FRW) metric of the universe as

$$
d s^{2}=-d t^{2}+a^{2}(t)\left[\frac{d r^{2}}{1-k r^{2}}+r^{2}\left(d \theta^{2}+\sin ^{2} \theta d \phi^{2}\right)\right]
$$


where $k(=0, \pm 1)$ is the curvature scalar and $a(t)$ is the scale factor. From equation $(3)$, we get

$$
K=\frac{3 \beta H^{2}}{M^{2}}
$$

where $H\left(=\frac{\dot{a}}{a}\right)$ is Hubble parameter and the coefficient $\beta$ is expressed as $\beta=c_{1}+3 c_{2}+c_{3}$ [43, 44] and it is constant. From eq. (4), we get the modified Friedmann equations (for flat universe $k=0$ ) for Einstein-Aether gravity as in the following [42, 43] form

$$
\beta\left(-F^{\prime}+\frac{F}{2 K}\right) H^{2}+H^{2}=\frac{8 \pi G}{3} \rho
$$

and

$$
\beta \frac{d}{d t}\left(H F^{\prime}\right)-2 \dot{H}=8 \pi G(\rho+p)
$$

Now, if $\rho_{E A}$ and $p_{E A}$ are considered as the effective energy density and pressure governed by the Einstein-Aether gravity, then we can write the eqs. (10) \&(11) in the following form:

$$
H^{2}=\frac{1}{3} \rho+\frac{8 \pi G}{3} \rho_{E A}
$$

and

$$
-2 \dot{H}=8 \pi G(\rho+p)+\left(\rho_{E A}+p_{E A}\right)
$$

where the effective energy density and pressure for Einstein-Aether gravity sector are given by (choosing $8 \pi G=1$ )

$$
\rho_{E A}=3 \beta H^{2}\left(F^{\prime}-\frac{F}{2 K}\right)
$$

and

$$
p_{E A}=-3 \beta H^{2}\left(F^{\prime}-\frac{F}{2 K}\right)-\beta\left(\dot{H} F^{\prime}+H \dot{F}^{\prime}\right)
$$

At this point we want to discuss some special cases due to the power law form of the function $F(K)$. It can be noted that the modified Friedmann equations (10) \& (11) can be rewritten as [44]

$$
\left[1-\beta \sqrt{K} \frac{d}{d K}\left(\frac{F}{\sqrt{K}}\right)\right] H^{2}=\frac{8 \pi G}{3} \rho
$$

and

$$
\frac{d}{d t}\left[-2 H+\beta H F_{K}\right]=8 \pi G(\rho+p)
$$

and if we consider $F(K)=\gamma K^{n}$ where $\gamma$ is a constant then eq. (16) become

$$
\left[1+\epsilon\left(\frac{H}{M}\right)^{2(n-1)}\right] H^{2}=\frac{8 \pi G}{3} \rho
$$

where $\epsilon \equiv \frac{1}{6}(1-2 n) \gamma 3^{n} \beta^{n}$ and also from eq. (18) we have

$$
\gamma=\frac{6\left(\Omega_{m}-1\right)}{(1-2 n) 3^{n} \beta^{n}}\left(\frac{M}{H_{0}}\right)^{2(n-1)}
$$

where $\Omega_{m}=8 \pi G \rho_{0} / 3 H_{0}^{2}$ and $H_{0}$ is the present value of Hubble parameter. Let us now consider some particular value of $n$ : If $n=1$, we have $\epsilon=-\gamma \beta / 2$ and Newton's constant is rescaled by a factor $1 /(1+\epsilon)$ [45] ; If $n=1 / 2$, then we have the unchanged Friedmann equation as $\epsilon=0$ and in this scenario there is no effect on the background cosmology; If $n=0$ we get the cosmological constant $\Lambda \simeq \operatorname{sign}(\gamma) M^{2}$. Therefore we can obtain different regimes depending on $n$ for that special scenario of power law form of the function $F(K)$.

In the following sections, we shall study the correspondence between Einstein-Aether gravity and other modified gravities like $f(T), f(R), f(G), f(R, T)$ and $f(R, G)$. 


\section{CORRESPONDENCE BETWEEN EINSTEIN-AETHER AND $f(T)$ GRAVITIES}

The action for the $f(T)$ gravity is given by 24,26$]$

$$
S_{T}=\frac{1}{2} \int d^{4} x \sqrt{-g}\left[f(T)+\mathcal{L}_{m}\right]
$$

where $T$ is the torsion scalar which can be chosen as $T=-6 H^{2}, f(T)$ is general differentiable function of the torsion and $8 \pi G=c=1$. The modified Friedmann equations for $f(T)$ gravity can be written as,

$$
H^{2}=\frac{1}{3}\left(\rho_{m}+\rho_{T}\right)
$$

and

$$
\dot{H}=-\frac{1}{2}\left(\rho_{m}+p_{m}+\rho_{T}+p_{T}\right)
$$

where $\rho_{T}$ and $p_{T}$ be the energy density and pressure in $f(T)$ gravity given by

$$
\rho_{T}=\frac{1}{2}\left(2 T f^{\prime}(T)-f(T)+6 H^{2}\right)
$$

and

$$
p_{T}=-\frac{1}{2}\left(-8 \dot{H} T f^{\prime \prime}(T)+(2 T-4 \dot{H}) f^{\prime}(T)-f(T)+4 \dot{H}+6 H^{2}\right)
$$

where prime and dot denote the derivatives w.r.t. $T$ and $t$ respectively.

Now we make correspondence between Einstein-Aether gravity and $f(T)$ gravity by equating their energy densities (14) and (23). For this purpose, we assume a simple power law form of $f(T)=f_{T 0} T^{m}(m>0)$, so we get the differential equation for $F(K)$ as in the form:

$$
\frac{d F}{d K}-\frac{F}{2 K}=A_{T} K^{m-1}
$$

where,

$$
A_{T}=\frac{(-1)^{m}}{\beta^{m}}(1-2 m) 2^{m-1} M^{2 m-2} f_{T 0}
$$

and from which we have

$$
F(K)=\frac{2 A_{T}}{2 m-1} K^{m}+B_{T} \sqrt{K}
$$

where $B_{T}$ is a constant. We observe that $F(K)$ is analytic function of $K$. So $F(K)$ can be reconstructed in the framework of $f(T)$ gravity. Also in figure 1, we have drawn the function $F(K)$ for different positive values of $m$. For, $m=2$, the function $F(K)$ first increases to some upper bound and then decreases. But for $m=3$ and 4 , the function $F(K)$ increases always.

\section{CORRESPONDENCE BETWEEN EINSTEIN-AETHER AND $f(R)$ GRAVITIES}

The action of $f(R)$ gravity with matter is given in the four-dimensional flat space-time by [21, 23],

$$
S_{R}=\int d^{4} x \sqrt{-g}\left[\frac{R+f(R)}{2}+\mathcal{L}_{m}\right]
$$

where $R$ is replaced with $f(R)$ to generalized the usual Einstein-Hilbert action, where $f(R)$ is an analytic function of $R$ and $\mathcal{L}_{m}$ is the matter Lagrangian. Also in this flat space-time $(k=0)$, the Ricci scalar is given by $R=6 \dot{H}+12 H^{2}$ and choosing $8 \pi G=c=1$. 


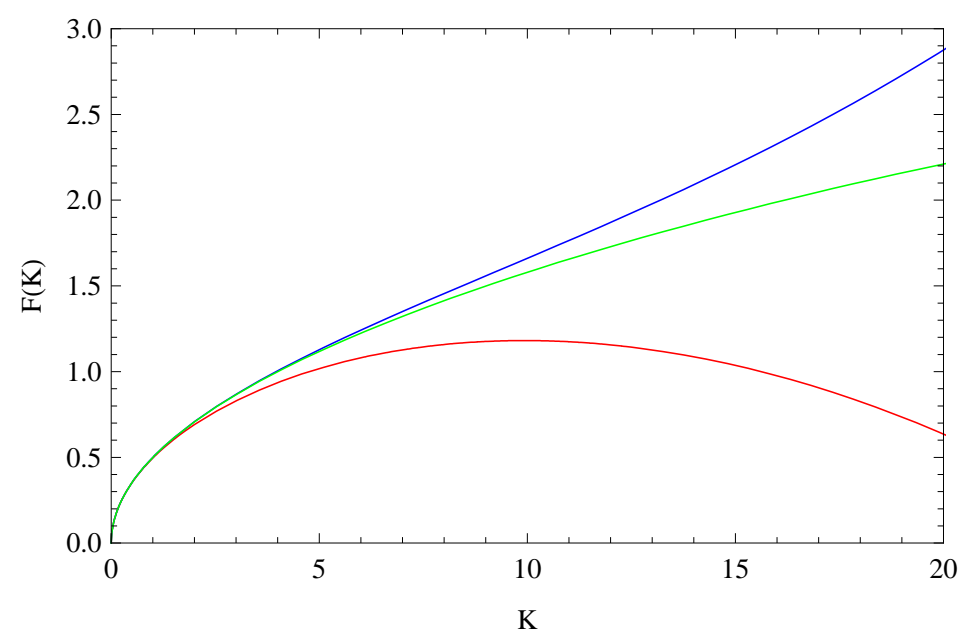

Fig.1

Fig. 1 shows the variation of reconstructed $F(K)$ against $K$ from $f(T)$ gravity theory where $M=0.01, \beta=0.1, B=0.5, f_{T 0}=1$ and $m=2$ (Red line), $m=3$ (Blue line), $m=4$ (Green line).

The gravitational field equations in flat $(k=0)$ space-time are given by

$$
3 H^{2}=\rho_{m}+\rho_{R}
$$

and

$$
2 \dot{H}+3 H^{2}=-\left(p_{m}+p_{R}\right)
$$

where $\rho_{m}$ being the energy density and $p_{m}$ is the pressure of matter and

$$
\rho_{R}=-\frac{1}{2} f(R)+3\left(\dot{H}+H^{2}\right) f^{\prime}(R)-18\left(4 H^{2} \dot{H}+H \ddot{H}\right) f^{\prime \prime}(R)
$$

and

$$
p_{R}=\frac{1}{2} f(R)-\left(\dot{H}+3 H^{2}\right) f^{\prime}(R)+6\left(8 H^{2} \dot{H}+6 H \ddot{H}+4 \dot{H}^{2}+\dddot{H}\right) f^{\prime \prime}(R)+36(\ddot{H}+4 H \dot{H})^{2} f^{\prime \prime \prime}(R)
$$

where prime and dot denote the derivatives w.r.t. $R$ and $t$. Now we make correspondence between Einstein-Aether gravity theory and $f(R)$ gravity theory by equating their energy densities (14) and (31). For this purpose, we assume

$$
f(R)=f_{R 0} R^{\mu}
$$

where $f_{R 0}$ and $\mu>0$ are constants. Again, we assume the power law form of the scale factor in the form $a=a_{0} t^{n}$, where, $a_{0}$ and $n$ are constants. For accelerating phase of the universe, we must have $n>1$, because the deceleration parameter $q$ must be negative. Now if we put the power law form of scale factor and equation (33) into equations (28) -(32), we get the relations between the unknown constants, which are not important for our present study. So we get the differential equation in the form

$$
\frac{d F}{d K}-\frac{F}{2 K}=\frac{A_{R} M^{2 \mu-2}}{3^{\mu} \beta^{\mu} n^{2 \mu}} K^{\mu-1}
$$

and from which we have

$$
F(K)=\left(\frac{A_{R} M^{2 \mu-2}}{3^{\mu} \beta^{\mu} n^{2 \mu}}\right)\left(\frac{K^{\mu}}{\mu-\frac{1}{2}}\right)+B_{R} \sqrt{K}
$$




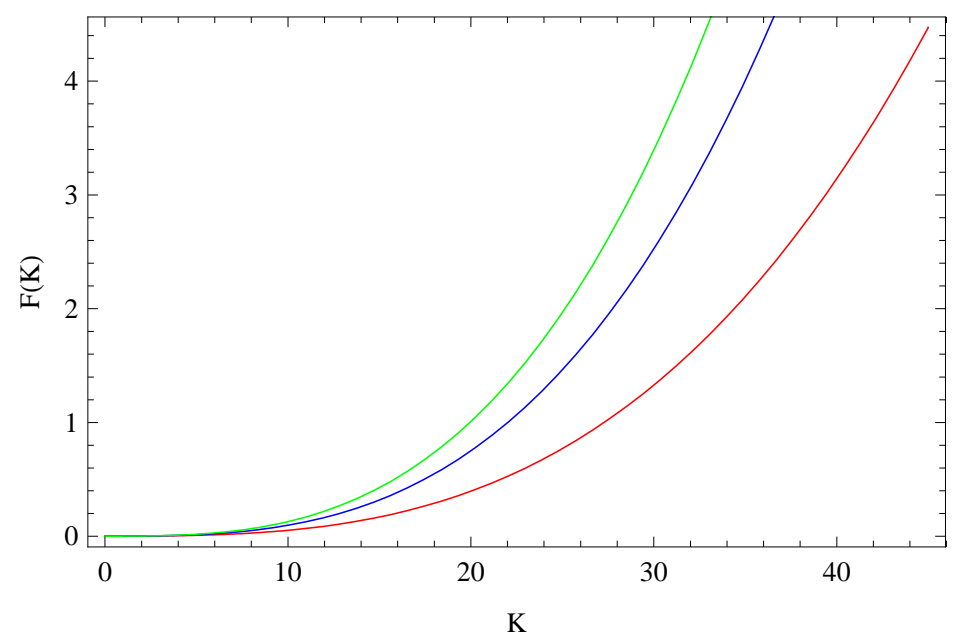

Fig.2

Fig. 2 shows the variation of reconstructed $F(K)$ against $K$ from $f(R)$ gravity theory where $n=3, M=0.01, \beta=0.1, B=0.001, f_{R 0}=1$ and $\mu=4$ (Red line), $\mu=5$ (Blue line), $\mu=6$ (Green line).

where we consider

$$
A_{R}=2^{\mu-1} 3^{\mu} n^{\mu} f_{R 0}\left(-1+\mu-2 \mu^{2}+(2+\mu) n\right)(1-2 n)^{\mu-1}
$$

and $B_{R}$ is arbitrary constant. We observe that $F(K)$ is analytic function of $K$ for $\mu \neq \frac{1}{2}$. So $F(K)$ can be reconstructed in the framework of $f(R)$ gravity. Also in figure 2, we have drawn the function $F(K)$ for different positive values of $\mu$. For $\mu=4,5$ and 6 , the function $F(K)$ increases always.

\section{CORRESPONDENCE BETWEEN EINSTEIN-AETHER AND $f(G)$ GRAVITIES}

The action for the $f(G)$ gravity is given by [27, 29],

$$
S_{G}=\frac{1}{2} \int d^{4} x \sqrt{-g}\left[\frac{1}{2} R+f(G)+\mathcal{L}_{m}\right]
$$

where $R$ is the Ricci scalar curvature and $\mathcal{L}_{m}$ is the matter Lagrangian. Here $f(G)$ is an arbitrary differentiable function of $G$ and $G$ is generally defined as $G=R_{\alpha \beta \gamma \delta} R^{\alpha \beta \gamma \delta}-4 R_{\alpha \beta} R^{\alpha \beta}+R^{2}$, where $R_{\alpha \beta}$ and $R_{\alpha \beta \gamma \delta}$ are Ricci and Riemann curvature tensors, respectively. Now, the variation of the action $S$, given by eq. (37) with respect to the metric tensor $g_{\alpha \beta}$ is given as follows [27, 28]:

$$
\begin{gathered}
0=\frac{1}{2}\left(-R^{\alpha \beta}+\frac{1}{2} g^{\alpha \beta} R\right)+T^{\alpha \beta}+\frac{1}{2} g^{\alpha \beta} f(G)-2 f^{\prime}(G) R R^{\alpha \beta}+4 f^{\prime}(G) R_{\gamma}^{\alpha} R^{\beta \gamma}-2 f^{\prime}(G) R^{\alpha \gamma \delta \tau} R_{\gamma \delta \tau}^{\beta} \\
+4 f^{\prime}(G) R^{\alpha \gamma \delta \beta} R_{\gamma \delta}+2\left(\nabla^{\alpha} \nabla^{\beta} f^{\prime}(G)\right) R-2 g^{\alpha \beta}\left(\nabla^{2} f^{\prime}(G)\right) R-4\left(\nabla_{\gamma} \nabla^{\alpha} f^{\prime}(G)\right) R^{\beta \gamma} \\
-4\left(\nabla_{\gamma} \nabla^{\beta} f^{\prime}(G)\right) R^{\alpha \gamma}+4\left(\nabla^{2} f^{\prime}(G)\right) R^{\alpha \beta}+4 g^{\alpha \beta}\left(\nabla_{\gamma} \nabla_{\delta} f^{\prime}(G)\right) R^{\gamma \delta}-4\left(\nabla_{\gamma} \nabla_{\delta} f^{\prime}(G)\right) R^{\alpha \gamma \beta \delta}
\end{gathered}
$$

Now the metric of flat Friedmann-Robertson-Walker(FRW) universe is defined as

$$
d s^{2}=-d t^{2}+a(t)^{2} \sum_{i=1}^{3}\left(d x^{i}\right)^{2}
$$


where $a(t)$ is the scale factor of the cosmic time $t$. From Eq. (39) with the help of Eq.(38) we have

$$
0=-f(G)+G f^{\prime}(G)-24 H^{3} \dot{G} f^{\prime \prime}(G)-6 H^{2}+2 \rho_{m}
$$

and

$$
0=f(G)-G f^{\prime}(G)+16 H^{3} \dot{G} f^{\prime \prime}(G)+16 H \dot{H} \dot{G} f^{\prime \prime}(G)+8 H^{2}\left(\ddot{G} f^{\prime \prime}(G)+\dot{G}^{2} f^{\prime \prime \prime}(G)\right)+2\left(2 \dot{H}+3 H^{2}\right)+2 p_{m}
$$

Therefore the modified Fridmann equations can be rewritten as,

$$
3 H^{2}=\rho_{m}+\rho_{G}
$$

and

$$
-\left(2 \dot{H}+3 H^{2}\right)=p_{m}+p_{G}
$$

where

$$
\rho_{G}=\frac{1}{2}\left[-f(G)+G f^{\prime}(G)-24 H^{3} \dot{G} f^{\prime \prime}(G)\right]
$$

and

$$
p_{G}=\frac{1}{2}\left[f(G)-G f^{\prime}(G)+16 H^{3} \dot{G} f^{\prime \prime}(G)+16 H \dot{H} \dot{G} f^{\prime \prime}(G)+8 H^{2}\left(\ddot{G} f^{\prime \prime}(G)+\dot{G}^{2} f^{\prime \prime \prime}(G)\right)\right]
$$

where prime and dot denote the derivatives w.r.t. $G$ and $t$ respectively and the Gauss-Bonnet invariant $G=24 H^{2}\left(H^{2}+\dot{H}\right)$.

Now we make correspondence between Einstein-Aether gravity theory and $f(G)$ gravity theory by equating their energy densities (14) and (44). For this purpose, we assume

$$
f(G)=f_{G 0} G^{\nu}
$$

where $f_{G 0}$ and $\nu>0$ are constants. Again, we assume the power law form of the scale factor in the form $a=a_{0} t^{n}$, where, $a_{0}$ and $n$ are constants. So we get the differential equation in the form

$$
\frac{d F}{d K}-\frac{F}{2 K}=A_{G} K^{\nu-1}
$$

and from which we have the solution

$$
F(K)=\frac{2 A_{G}}{4 \nu-1} K^{2 \nu}+B_{G} \sqrt{K}
$$

where we consider

$$
A_{G}=\frac{\left(\frac{8}{3}\right)^{\nu} f_{G 0}(-1+\nu)(-1+4 \nu+n)\left(\frac{M^{4}(-1+n)}{n \beta^{2}}\right)^{\nu}}{2 M^{2}(-1+n)}
$$

and $B_{G}$ is arbitrary constant. We observe that $F(K)$ is analytic function of $K$ for $\nu \neq \frac{1}{4}$. So $F(K)$ can be reconstructed in the framework of $f(G)$ gravity. Also in figure 3, we have drawn the function $F(K)$ for different positive values of $\nu$. For, $\nu=2$, the function $F(K)$ first increases to some upper bound and then decreases. But for $\nu=3$ and 4 , the function $F(K)$ increases always.

\section{CORRESPONDENCE BETWEEN EINSTEIN-AETHER AND $f(R, T)$ GRAVITIES}

The action for the $f(R, T)$ gravity is given by [30, 31, 47],

$$
S=\frac{1}{2} \int d^{4} x \sqrt{-g}\left[\frac{f(R, T)}{16 \pi G}+\mathcal{L}_{m}\right]
$$




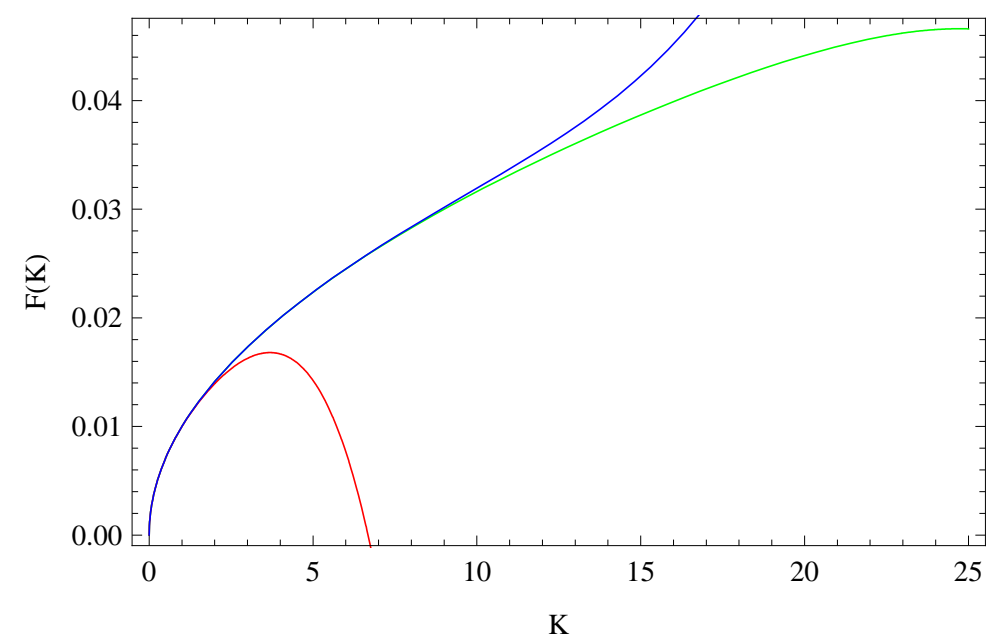

Fig.3

Fig.3 shows the variation of reconstructed $F(K)$ against $K$ from $f(G)$ gravity theory where $n=0.1, M=0.01, \beta=0.1, B_{G}=0.01$ and $\nu=2$ (Red line), $\nu=3$ (Blue line), $\nu=4$ (Green line).

where $\mathcal{L}_{m}$ denotes the matter contents of the universe. In FRW background, the gravitational field equation are given by

$$
\begin{gathered}
3 H^{2}=8 \pi G_{e f f}\left(\rho_{m}+\rho_{R T}\right) \\
\dot{H}=-4 \pi G_{e f f}\left(\rho_{m}+p_{m}+\rho_{R T}+p_{R T}\right)
\end{gathered}
$$

where

$$
\begin{gathered}
\rho_{R T}=3 H^{2} \frac{f_{T}-f_{R}+1}{1+f_{T}}+\frac{\frac{1}{2}\left(R f_{R}-f\right)-3 H \dot{R} f_{R R}}{1+f_{T}} \\
p_{R T}=\frac{1}{1+f_{T}}\left[-\frac{1}{2}\left(R f_{R}-f\right)+2 H \dot{R} f_{R R}+\ddot{R} f_{R R}+\dot{R}^{2} f_{R R R}\right]
\end{gathered}
$$

and

$$
G_{e f f}=\frac{1}{f_{R}(R, T)}\left(G+\frac{f_{T}(R, T)}{8 \pi}\right)
$$

is the effective gravitational matter dependent coupling in $f(R, T)$ gravity Here $\rho_{R T}$ and $p_{R T}$ are the energy density and pressure of dark energy and choosing $8 \pi G=c=1$. Here, the prime denotes the non-equilibrium description of the field equations. Now we make correspondence between Einstein-Aether gravity theory and $f(R, T)$ gravity theory by equating their energy densities (14) and (53). For this purpose, we assume

$$
f(R, T)=d_{1} R^{\mu_{1}}+d_{2} T^{\mu_{2}}
$$

where $d_{1}, d_{2}, \mu_{1}>0$ and $\mu_{2}>0$ are constants. Again, we assume the power law form of the scale factor in the form $a=a_{0} t^{n}$, where, $a_{0}$ and $n$ are constants. So we get the differential equation in the form

$$
\begin{aligned}
\frac{d F}{d K}- & \frac{F}{2 K}=\frac{1}{\beta}+\left(\frac{d_{2} \mu_{2}}{\beta}\left(-6 n^{2}\right)^{\mu_{2}-1}-\frac{d_{1} \mu_{1}}{\beta}(6 n(6 n-1))^{1-\mu_{1}}-\frac{d_{2}}{6 \beta n^{2}}\left(-6 n^{2}\right)^{\mu_{2}}\right)\left(\frac{3 \beta n^{2}}{M^{2}}\right)^{1-\mu_{2}} K^{\mu_{2}-1} \\
& +\left(\frac{\left(\mu_{1}-1\right)}{6 \beta n^{2}}(6 n(6 n-1))^{\mu_{1}}+\frac{12 d_{1} \mu_{1}(2 n-1)\left(\mu_{1}-1\right)}{\beta}(6 n(6 n-1))^{\mu_{1}-2}\right)\left(\frac{3 \beta n^{2}}{M^{2}}\right)^{1-\mu_{1}} K^{\mu_{1}-1}
\end{aligned}
$$




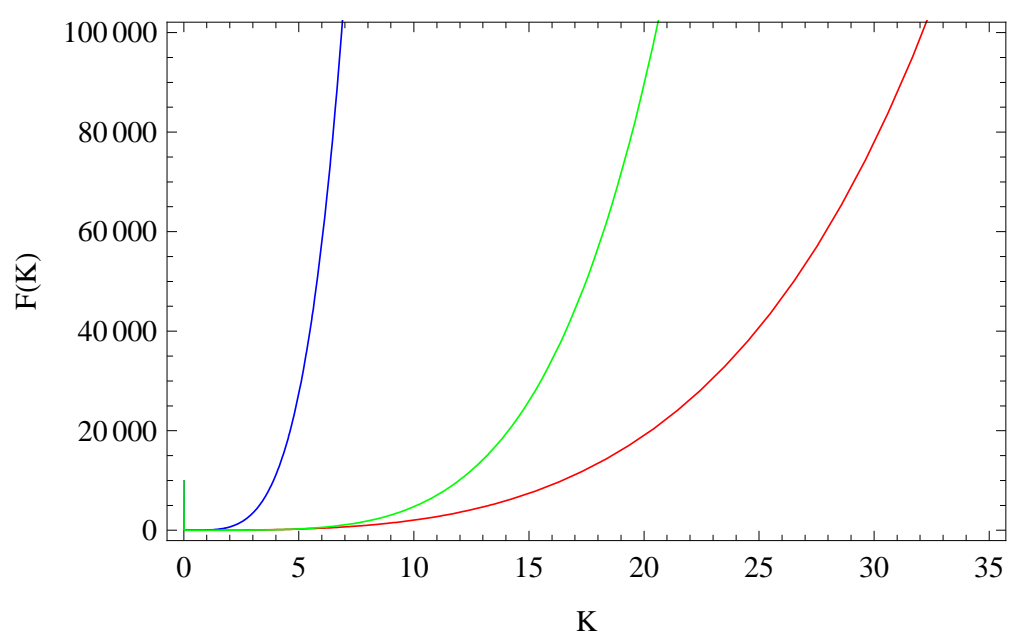

Fig.4

Fig.4 shows the variation of reconstructed $F(K)$ against $K$ from $f(R, T)$ gravity theory where $n=5, M=2, \beta=1, B_{R T}=10$ and $\mu_{1}=3, \mu_{2}=2, d_{1}=0.01, d_{2}=0.001$ (Red line), $\mu_{1}=4, \mu_{2}=2, d_{1}=0.01, d_{2}=0.001$ (Blue line), $\mu_{1}=4, \mu_{2}=2, d_{1}=0.0001, d_{2}=0.001$ (Green line).

and from which we have (for $\mu_{2}=2$ )

$$
\begin{gathered}
F(K)=\frac{B_{R T}}{\sqrt{K}}-\frac{1}{8 \sqrt{K} M^{2}(-1+2 n) \beta d_{2}^{3 / 2}\left(M+2 M \mu_{1}\right)}\left((-1+2 n) \beta^{3 / 2} \tanh ^{-1}\left[\frac{2 \sqrt{K} M \sqrt{d_{2}}}{\sqrt{\beta}}\right]\left(1+2 \mu_{1}\right)-\right. \\
2 \sqrt{K} M(-1+2 n) \beta \sqrt{d_{2}}\left(1+2 \mu_{1}\right)-8 \sqrt{K} M d_{2}^{3 / 2}\left(K M^{2}(-1+2 n)\left(1+2 \mu_{1}\right)+2^{\mu_{1}}\left(\frac{K M^{2}(-1+2 n)}{n \beta}\right)^{\mu_{1}} \beta\right. \\
\left.\left.\times{ }_{2} F_{1}\left[\frac{1}{2}+\mu_{1}, 1, \frac{3}{2}+\mu_{1}, \frac{4 K M^{2} d_{2}}{\beta}\right] d_{1}\left(1-2 n+(-3+n) \mu_{1}+2 \mu_{1}^{2}\right)\right)\right)
\end{gathered}
$$

and $B_{R T}$ is arbitrary constant. Now $F(K)$ can be reconstructed in the framework of $f(R, T)$ gravity. Also in figure 4 , we have drawn the function $F(K)$ for different positive values of $d_{1}, d_{2}, \mu_{1}$ and $\mu_{2}$ and we see that the function $F(K)$ increases always.

\section{CORRESPONDENCE BETWEEN EINSTEIN-AETHER AND $f(R, G)$ GRAVITIES}

The action of $f(R, G)$ gravity is given by [32, 33],

$$
S_{R G}=\int d^{4} x \sqrt{-g}\left[\frac{1}{2} f(R, G)+\mathcal{L}_{m}\right]
$$

In the case of flat FRW Universe, the Einstein's first field equation is

$$
3 H^{2}=\rho_{m}+\rho_{R G}
$$

The contribution of energy density for $f(R, G)$ gravity is given by

$$
\rho_{R G}=3 H^{2}+\frac{1}{2}\left(G f_{G}-f(R, G)-24 H^{3} f_{G t}\right)+3\left(\dot{H}+H^{2}\right) f_{R}-3 H f_{R t}
$$

where $f(R, G)$ is a more general kind of modified Gauss-Bonnet gravity and suffix denote the partial derivatives. Now we make correspondence between Einstein-Aether gravity theory and $f(R, G)$ gravity theory by equating their energy densities (14) and (61). For this purpose, we assume

$$
f(R, G)=d_{3} R^{\mu_{3}}+d_{4} G^{\mu_{4}}
$$




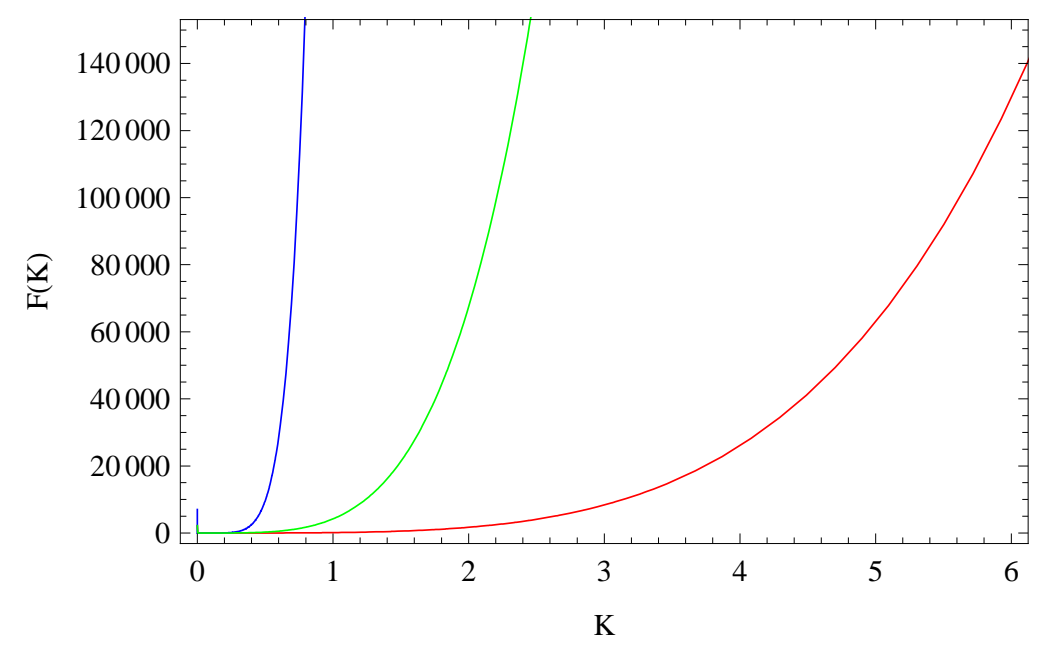

Fig.5

Fig.5 shows the variation of reconstructed $F(K)$ against $K$ from $f(R, G)$ gravity theory where $n=5, M=2, \beta=0.1, B_{R}=1$ and $\mu_{3}=3, \mu_{4}=2, d_{3}=0.0001, d_{4}=0.0001$ (Red line), $\mu_{3}=3, \mu_{4}=3, d_{3}=0.0001, d_{4}=0.0001$ (Blue line), $\mu_{3}=5, \mu_{4}=2, d_{3}=0.0001, d_{4}=0.0001$ (Green line).

where $d_{3}, d_{4}, \mu_{3}>0$ and $\mu_{4}>0$ are constants. Again, we assume the power law form of the scale factor in the form $a=a_{0} t^{n}$, where, $a_{0}$ and $n$ are constants. So we get the differential equation in the form

$$
\begin{aligned}
& \frac{d F}{d K}-\frac{F}{2 K}=\frac{1}{2 K M^{2}}\left(\frac{2 K M^{2}}{\beta}+\frac{2^{\mu_{3}}\left(\frac{K M^{2}(-1+2 n)}{n \beta}\right)^{\mu_{3}} d_{3}\left(1-2 n+\mu_{3}\left(-3+n+2 \mu_{3}\right)\right)}{-1+2 n}\right. \\
& \left.+\frac{\left(\frac{8}{3}\right)^{\mu_{4}}\left(\frac{K^{2} M^{4}(-1+n)}{n \beta^{2}}\right)^{\mu_{4}} d_{4}\left(-1+\mu_{4}\right)\left(-1+n+4 \mu_{4}\right)}{-1+n}\right)
\end{aligned}
$$

and from which we have

$$
\begin{aligned}
& F(K)=\frac{B_{R G}}{\sqrt{K}}+\frac{3^{-1-\mu_{4}}}{M^{2}\left(1-3 n+2 n^{2}\right) \beta}\left(2 \times 3^{\mu_{4}} K M^{2}\left(1-3 n+2 n^{2}\right)\right. \\
& +\frac{2^{\mu_{3}} 3^{1+\mu_{4}}(-1+n)\left(\frac{K M^{2}(-1+2 n)}{n \beta}\right)^{\mu_{3}} \beta d_{3}\left(1-2 n+(-3+n) \mu_{3}+2 \mu_{3}^{2}\right)}{1+2 \mu_{3}}-\frac{3}{1+4 \mu_{4}}\left(\frac{K^{2} M^{4}(-1+n)}{n \beta^{2}}\right) \\
& \left.\quad \times \beta d_{4}\left(8^{\mu_{4}}-38^{\mu_{4}} n+2^{1+3 \mu_{4}} n^{2}+\left(-58^{\mu_{4}}+118^{\mu_{4}} n-2^{1+3 \mu_{4}} n^{2}\right) \mu_{4}-2^{2+3 \mu_{4}}(-1+2 n) \mu_{4}^{2}\right)\right)
\end{aligned}
$$

and $B_{R G}$ is arbitrary constant. Now $F(K)$ can be reconstructed in the framework of $f(R, G)$ gravity. Also in figure 5 , we have drawn the function $F(K)$ for different positive values of $d_{3}, d_{4}, \mu_{3}$ and $\mu_{4}$ and we see that the function $F(K)$ increases always.

\section{STABILITY ANALYSIS OF RECONSTRUCTED EINSTEIN-AETHER GRAVITY MODELS}

In this section, we have examined the stability of each reconstructed Einstein-Aether gravity models from $f(T)$, $f(R), f(G), f(R, T)$ and $f(R, G)$. In this connection, the most important quantity is that the square speed of sound which is denoted as $v_{s}^{2}$ and defined as a ratio of the effective pressure and energy densities i.e., $v_{s}^{2}=\frac{\dot{p}}{\dot{\rho}}$. The sign of $v_{s}^{2}$ 
plays a very vital role for stability analysis of a background evolution of cosmic models. It is well known that the model is stable if $v_{s}^{2}>0$ and if $v_{s}^{2}<0$ implies that the model is classically unstable [48]. In 2008, Kim et al. [49] found that $v_{s}^{2}$ for agegraphic DE is always negative which leads to classically instability of that model. Recently many researchers are using this methods to analyzed the stability of that models on which they worked and of which some authors 50 53] have reached to a conclusion that HDE, ADE, NADE, Chaplygin gas, holographic Chaplygin, holographic $f(T)$, holographic $f(G)$, new agegraphic $f(T)$, new agegraphic $f(G)$ models are classically unstable because square speed of sound is negative i.e., $v_{s}^{2}<0$ throughout the evolution of the universe. Here we consider

$$
v_{s}^{2}=\frac{\dot{p}_{E A}}{\dot{\rho}_{E A}}
$$

and plot $v_{s}^{2}$ versus $t$ by taking the power-law scale factor in the each reconstructed Einstein-Aether Gravity model and fig. 6-10 show the variation of $v_{s}^{2}$ with $t$ for those above cases. In the reconstructed Einstein-Aether gravity model from $f(T), f(R)$ and $f(G)$ model we observed (Figs. 6-8) that the square speed of sound remains positive for the present and future epoch, that implies reconstructed Einstein-Aether Gravity model from those scenarios with power-law scale factor are classically stable. Whereas in the case of $f(R, T)$, we found that $v_{s}^{2}$ remains negative for the present and future epoch which means reconstructed Einstein-Aether Gravity model from $f(R, T)$ scenario with power-law scale factor is classically unstable. But when we considered the $f(R, G)$ model, we shown that $v_{s}^{2}$ remains positive as well as negative for a certain period of time $t$, which entailed that the Einstein-Aether Gravity model reconstructed from $f(R, G)$ is classically stable for a certain period of time and unstable for remaining epoch.

\section{DISCUSSIONS AND CONCLUDING REMARKS}

We briefly describe one of the modified gravity named as Einstein-Aether gravity. In FRW model, the Friedmann equations for this gravity have been discussed. We find the effective energy density and pressure for Einstein-Aether gravity. We have analyzed the correspondence between Einstein-Aether gravity and other modified gravities like $f(T), f(R), f(G), f(R, T)$ and $f(R, G)$ gravities by comparing their energy densities. For this purpose, we have chosen the scale factor in power law form. Next we have found the free function $F(K)$ for Einstein-Aether gravity (where $K$ is proportional to $H^{2}$ ) in term of $K$ in the framework of the above modified gravity models separately. Thus the reconstruction is possible for these models. For $f(T), f(R)$ and $f(G)$ models, we have found the function $F(K)$ is analytic function in term of $K$. The nature of $F(K)$ vs $K$ have been shown graphically for every cases. For $f(T)$ gravity model, we have drawn the function $F(K)$ for different positive values of $m$ in figure 1 . For, $m=2$, the function $F(K)$ first increases to some upper bound and then decreases. But for $m=3$ and 4 , the function $F(K)$ increases always. For $f(R)$ gravity model, we have drawn the function $F(K)$ for different positive values of $\mu$ in figure 2. For $\mu=4,5$ and 6 , the function $F(K)$ always increases. For $f(G)$ gravity model, we have drawn the function $F(K)$ for different positive values of $\nu$ in figure 3. For, $\nu=2$, the function $F(K)$ first increases to some upper bound and then decreases. But for $\nu=3$ and 4 , the function $F(K)$ also increases. In figures 4 and 5 , we have drawn the function $F(K)$ for different positive values of constants in our $f(R, T)$ and $f(R, G)$ gravity models respectively and we see that the function $F(K)$ increases for these models. Also we checked the stability of reconstructed Einstein-Aether Gravity model from $f(T), f(R), f(G), f(R, T)$ and $f(R, G)$ models through the square speed of sound $v_{s}^{2}$ and fig.

6-10 show the variation of $v_{s}^{2}$ with $t$ for those cases. Finally, as results, we shown that Einstein-Aether Gravity model is classically stable when it is reconstructed from $f(T), f(R)$ and $f(G)$ models and classically unstable when it is reconstructed from $f(R, T)$ model and classically stable for a certain period of time as well as classically unstable for remaining epoch when it is reconstructed from $f(R, G)$ model.

\section{Acknowledgement:}

The authors are thankful to IUCAA, Pune, India for warm hospitality where part of the work was carried out.

[1] Gold, B., et al., 2009, Astrophys. J. Suppl. 180, 265.

[2] Nolta, M. R., et al., 2009, Astrophys. J. Suppl. 180, 296.

[3] Bachall, N. A., et al., 1999, Science 284, 1481. 


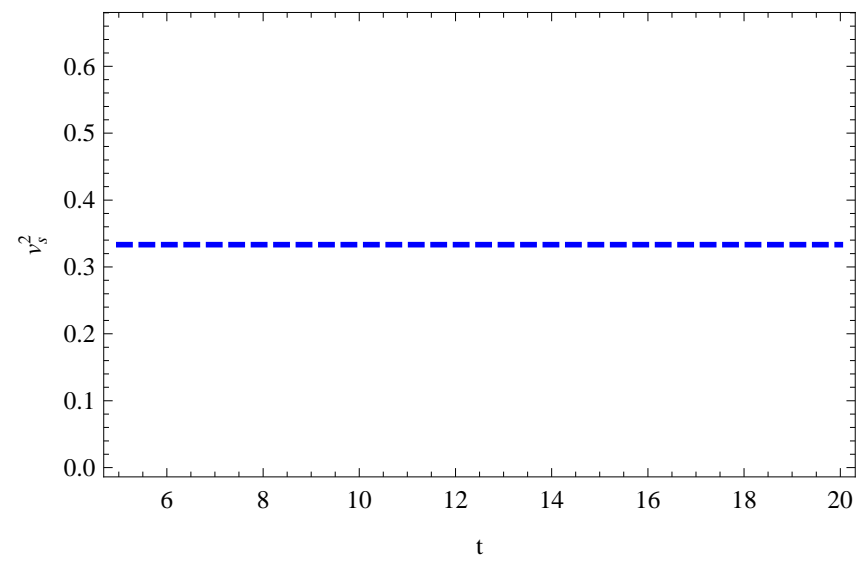

Fig.6

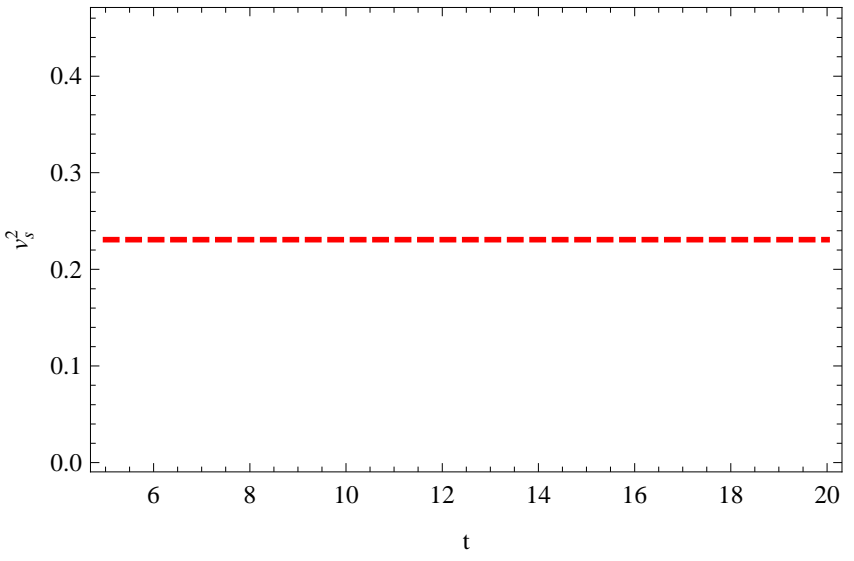

Fig.7

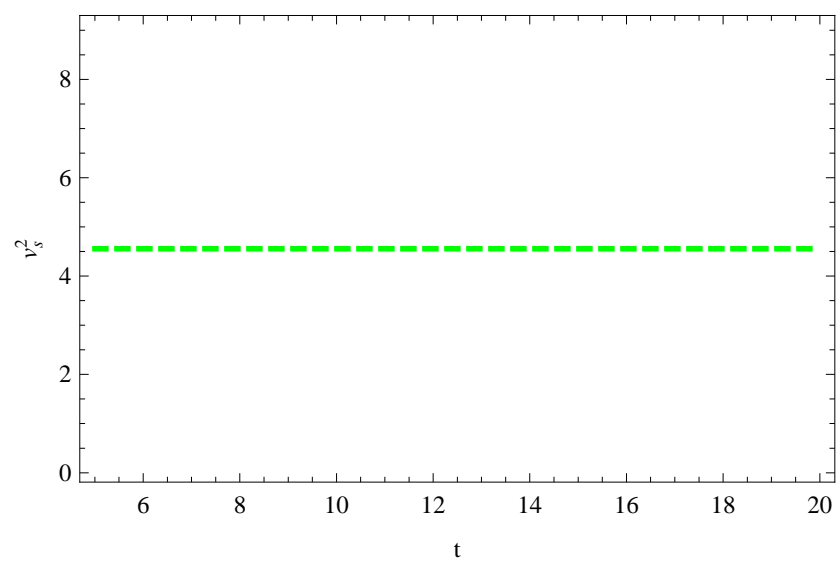

Fig.8

Figs. 6-8 show the variations of $v_{s}^{2}$ with $t$ for the reconstructed Einstein-Aether theory from $f(T)$ gravity theory (blue, dashed line), from $f(R)$ gravity theory (red, dashed line), from $f(G)$ gravity theory (green, dashed line) respectively.

[4] Perlmutter, S. J., et al., 1999, ApJ. 517, 565.

[5] Perlmutter, S. J., et al., 1998, Nature 391, 51.

[6] Riess, A. G., et al.[Supernova Search Team Collaboration], 1998, Astron. J. 116, 1009.

[7] Riess, A. G., et al., 2004, Astrophys. J. 607, 665.

[8] Bennet, C., et al., 2000, Phys. Rev. Lett. 85, 2236.

[9] Spergel, D. N., et al., 2007, Astrophys. J. Suppl. Ser. 170, 377.

[10] Sahni, V., et al., 2006, Int. J. Mod. Phys. D 15, 2105.

[11] Seikel, M., et al., 2012, JCAP 06, 036.

[12] Clarkson, C., et al., 2010, Phys. Rev. Lett. 104, 211301.

[13] Liu, X. M., et al., 2011, Astrophys. Space Sci. 334, 203.

[14] Vollick, D. N., 2007, Phys. Rev. D 76, 124001.

[15] Briscese, F., et al., 2007, Phys. Lett. B 646, 105.

[16] Carroll, S. M., et al., 2004, Phys. Rev. D 70, 043528.

[17] Abdalla, M. C. B., et al., 2005, Class. Quantum Grav. 22, L35.

[18] Linder, E. V., 2010, Phys. Rev. D 81, 127301.

[19] Nojiri, S., et al., 2003, Phys. Rev. D 68, 123512.

[20] Nojiri, S., et al., 2007, Int. J. Geom. Methods Mod. Phys. 4, 115.

[21] Nojiri, S., et al., 2008, Phys. Lett. B 659, 821.

[22] Li, B. and Barrow, J. D., 2007, Phys. Rev. D 75, 084010.

[23] Nojiri, S., Odintsov, S. D., 2006, Phys. Rev. D 74, 086005. 


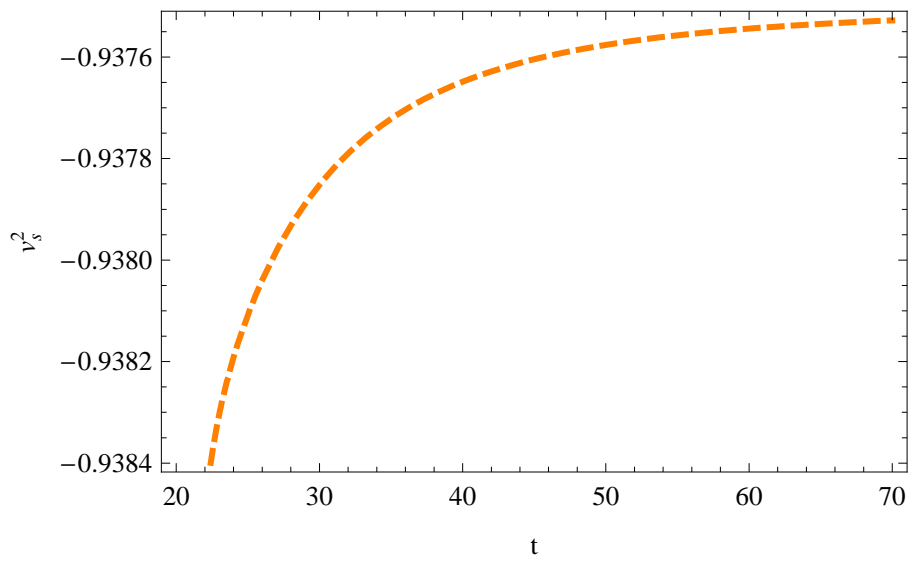

Fig.9

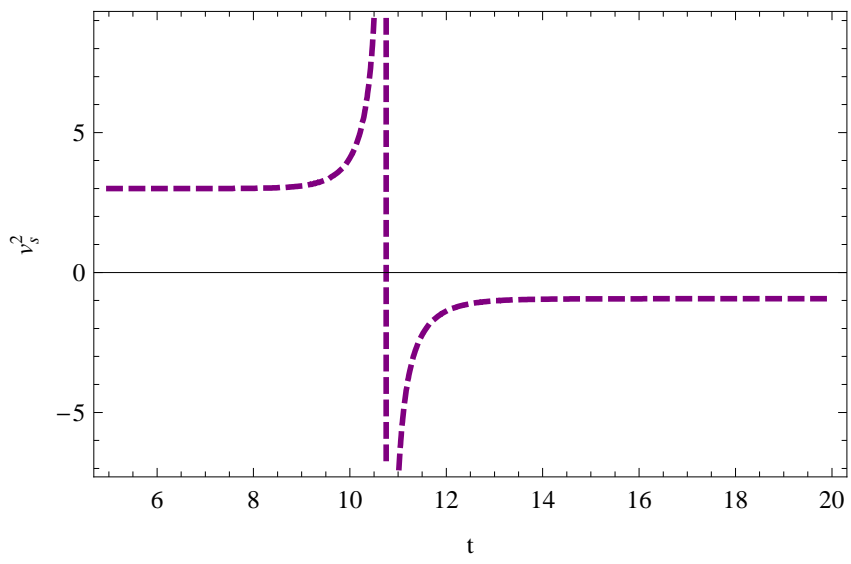

Fig. 10

Figs. 9-10 show the variations of $v_{s}^{2}$ with $t$ for the reconstructed Einstein-Aether theory from $f(R, T)$ gravity theory (orange, dashed line), from $f(R, G)$ gravity theory (purple, dashed line) respectively.

[24] Li, B., et al., 2011, Phys. Rev. D 83, 104017.

[25] Myrzakulov, R., 2011, Eur. Phys. J. C 71, 1752.

[26] Wu P., et al., 2011, Eur. Phys. J. C 71, 1552.

[27] Rastkar, A. R., et al., 2012, Astrophys. Space Sci. 337, 487.

[28] Nojiri, S., et al., 2005, Phys. Rev. D 71, 123509.

[29] Nojiri, S., Odintsov, S.D., 2005, Phys. Lett. B 631, 1.

[30] Harko, T., et al., 2011, Phys. Rev. D 84, 024020.

[31] Jamil, M., et al., 2012, Eur. Phys. J. C 72, 1999.

[32] Bamba, K., et al., 2010, Eur. Phys. J. C 67, 295.

[33] Myrzakulov, R., et al., 2013, Gen Relativ Gravit 45, 675.

[34] Setare, M. R., et al., 2008, Phys. Lett. B 670, 1.

[35] Liu, X. L., et al., 2009, Commun. Theor. Phys. 52, 761.

[36] Setare, M. R., 2010, Astrophys. Space Sci. 326, 27.

[37] Jamil, M., et al., 2010, JCAP 07, 028.

[38] Setare, M. R., et al., 2010, EPL 92, 49003.

[39] Jawad, A. et al., 2013 Astrophys. Space Sci. 344, 489.

[40] Debnath, U., 2013, arXiv:1311.0437[gr-qc].

[41] Jacobson, T., et al., 2001, Phys. Rev. D 64, 024028.

[42] Zlosnik, T. G., et al., 2007, Phys. Rev. D 75, 044017.

[43] Meng, X., et al., 2012, Phys. Lett. B 710, 493.

[44] Clifton, T., et al., 2012, Physics Reports 513, 1.

[45] Carroll, S. M. et al., 2004, Phys.Rev. D,70,123525.

[46] Jacobson, T., et al., 2004, Phys. Rev. D 70, 024003.

[47] Sharif, M. et al., 2012, JCAP, 03, 028.

[48] Myung, Y. S., 2007, Phys. Lett. B, 652, 223.

[49] Kim, K. Y.,Lee, H. W. and Myung, Y. S., 2008, Phys. Lett. B 660, 118.

[50] Jawad, A., Chattopadhyay, S. and Pasqua, A., 2013, Eur. Phys. J. Plus, 128, 88.

[51] Ebrahimi, E. and Sheykhi, A., 2011, Int. J. Mod. Phys. D 20, 2369.

[52] Sharif, M. and Jawad, A., 2012, Eur. Phys. C 722097.

[53] Setare, M. R., 2007, Phys. Lett. B 654, 1. 